\title{
Database development for additive manufacturing
}

\author{
Tracie Prater ${ }^{1}$
}

Received: 5 April 2016 / Accepted: 4 January 2017 / Published online: 21 February 2017

(C) Springer International Publishing Switzerland (outside the USA) 2017

\begin{abstract}
Additive manufacturing (AM) of metallics is a technology that is poised to revolutionize the way the aerospace industry manufactures propulsion hardware. The additive manufacturing laboratory at NASA Marshall Space Flight Center increasingly uses AM as a manufacturing technique to support development of lower cost propulsion systems. In a typical AM build, witness test coupons are built alongside additively manufactured parts or are excised from the parts themselves to determine the mechanical properties of those parts for use in design. These coupons undergo mechanical testing, resulting in a wealth of data that have yet to be archived or analyzed in a coherent form. The AM community faces many challenges with regard to data management and sharing of data to accelerate materials development and inform material discovery. This paper is a case study of development of an AM database and the analysis of alternatives conducted as part of its formulation. Segregation and organization of existing additive manufacturing data using the NASA-developed materials informatics system MAPTIS (Materials and Processing Technical Information System) is explored. Within the NASA-developed AM database, data can be organized and filtered by material type, AM process, and build parameters as well as in terms of mechanical test data (tensile, fatigue, fracture, surface finish, etc.). This work can serve as a model for how other organizations and research entities might utilize informatics to organize AM data and facilitate comparison of properties across AM builds as well as against conventionally manufactured materials. The
\end{abstract}

Tracie Prater

tracie.j.prater@nasa.gov

1 NASA Marshall Space Flight Center, Huntsville, AL 35812, USA archiving of build data in a database format makes it easier to explore interactions and correlations between factors that are of critical interest to the additive manufacturing community. An informatics approach also makes it possible to assess repeatability and scalability of AM processes across vendors and parts. The informatics work in this field strongly complements the development of standards and baseline material properties for additively manufactured materials and a rational path to flight certification for vendors and parts.

Keywords Additive manufacturing - Informatics . Databases $\cdot$ Materials development $\cdot$ Metallics $\cdot 3 D$ printing

\section{Introduction}

Although additive manufacturing technologies first emerged over two decades ago, the technology has become a high research priority for government agencies in recent years as the US government increasingly invests in initiatives aimed at revitalizing the manufacturing sector by creating "innovation in the products that are manufactured and the manufacturing systems themselves" [1]. Advanced manufacturing was recently identified as a top multi-agency R\&D priority by the Office of Management and Budget and the Office of Science and Technology Policy [1]. Similarly, the National Strategic Plan for Advanced Manufacturing calls for development of manufacturing tools and technologies that will benefit multiple sectors and promote future industry [2]. To facilitate collaboration, knowledge transfer, and data collection and management among multiple communities in both government and industry, the Obama administration established America Makes, a nonprofit 
organization comprised of over 100 companies, government agencies and academic institutions engaged in additive manufacturing research [3].

Additive manufacturing (AM) is the centerpiece of these efforts. The Materials and Processes Laboratory at NASA Marshall Space Flight Center in Huntsville, AL has been engaged in additive manufacturing research for over 20 years. The Marshall Center has state-of-the-art capabilities and immense technical expertise in numerous AM techniques, including selective laser melting (SLM), electron beam melting (EBM), and polymeric printing methods. As the technology has matured from a laboratory curiosity to a manufacturing method that can produce not only prototypes but production quality parts, the broader aerospace industry has adopted AM and integrated it into design processes. Boeing, SpaceX, Lockheed Martin, GE Aviation, and many other major aerospace companies are making substantial investments in AM technologies, as they see its potential to make space hardware more cost-effective, reduce the time from "art to part", detect problems earlier in the development cycle, reduce the number of welds, enable greater design flexibility, compress the design/build/test loop, and facilitate cost-effective low-batch production. The industry's new focus on additive manufacturing became apparent in 2012 when GE Aviation purchased Morris Technologies and its sister company Rapid Quality Manufacturing to produce parts for its LEAP engines used in commercial airliners [4].

The aerospace community, however, faces unique challenges that must be addressed before the potential economic benefits of AM can be realized. Chief among these is that there is currently no established path to certification for additively manufactured parts. Certification is controversial since AM materials and processes lack specifications which are comparable to controls established through the Society of Automotive Engineers (SAE) American Material Society (AMS) standards for conventionally manufactured materials. A draft specification for NASA hardware is in development [5]. For flight hardware, there is a critical need for material property characterization and an allowables development program. AM parts are process specific and standards are required to assess sensitivity of parts to base material (powder) composition, address machine variability, establish post-processing controls (i.e., heat treatment and surface treatment protocols), and ultimately ensure that AM products are safe and reliable prior to their deployment in crewed systems.

The primary barrier to important standards development, qualification, and certification efforts is not a lack of data. While the user community for AM is extremely large and extends beyond just the aerospace industry, the standards development community is comparatively small (but growing rapidly). American Society of Testing Materials committee F42 is one of the organizations leading the standards development effort. AM research often occurs in isolation and data sharing is impeded by ITAR (International Treaty of Arms Regulations) restrictions and concerns about proprietary data. In general, the private sector and academic institutions lack the financial incentive and resources to engage in the sort of data management work necessary to support certification efforts. Standards development for materials is typically undertaken by standards developing organizations (SDOs), which bring together experts from industry, academia, and government to develop voluntary consensus standards. NASA and other government organizations play a major role in providing data and the scientific foundation for standards development. Informatics represents the most efficient means to organize and manipulate data for this purpose.

Material informatics has the potential to address many of the aforementioned technologies and measurement challenges for additive manufacturing by providing a single source repository for AM data that can be used to characterize materials, explore the relationships between process variables and properties, disseminate validated materials property data to the modeling community, and aid in the development of material standards and protocols [6]. The Materials Selection and Control group within the Materials and Processes Laboratory at NASA Marshall has a long history of developing informatics tools to facilitate rational material selection and design, most notably MAPTIS. This system catalogs data related to mechanical and physical properties, environmental compatibility, flammability, toxic off-gassing in pressurized volumes, thermal vacuum stability, impact resistance in pressurized oxygen, space environmental effects, corrosion, and hydrogen embrittlement for over 50,000 unique materials [7]. While some AM test and materials data were previously archived in MAPTIS, data were not housed in a central repository or organized in a manner that can facilitate meaningful analysis [8]. This paper examines the potential of an informatics database to address technical challenges of AM, provides an overview of desired database capabilities, offers insight into preliminary database development using data from NASA MSFC's advanced manufacturing technology team, and presents a path forward for evolution and continual improvement of the AM tool.

\section{Overview of database capabilities}

The AM community faces challenges in data management, data sharing, and mining data efficiently to accelerate materials development and inform material discovery. 
Informatics has the potential to address many of the AM community's needs in the aforementioned areas. Practitioners, material researchers, and organizations undertaking standards development would be best served by an AM database with the following characteristics and capabilities:

1. Store and distribute previous data In most laboratories or production environments, an AM build is typically accompanied by a "witness sample", most often a round tensile specimen that is built simultaneously from the same powder and at the same conditions and process parameters as the AM part. This witness specimen undergoes destructive testing and the mechanical test data results are cataloged to ensure traceability. Depending on the throughput of the laboratory or production setting, there may thus be a large amount of archived data that may need to be imported into the database.

2. Catalog qualitative as well as quantitative data Not all data cataloged for AM builds are numerical. Information related to build identification, material pedigree, and systems equipment/calibration may be qualitative in nature. There may also be a need to upload test specimen drawings, chemical analysis reports, and microstructural images associated with AM builds. The database must be able to accommodate these inputs (which may take the form of multi-page documents or image files) as well as the traditional material property data.

3. Sophisticated filtering The large number of process variables associated with AM creates an expansive combinatorial space. An advanced filtering capability will thus be required to enable effective data mining, a capability which is key to reducing redundant research efforts. End-users of an AM database must be able to filter and segregate the data in ways that will facilitate comparative evaluation of AM builds and exploration of process/property relationships that are of critical interest to the AM community. The filtering scheme must be able to accommodate holes and gaps in data, as the attributes we wish to capture for future builds may be more extensive and/or different from those recorded up to this point. In this manner, filtering permits "old" and "new" data to be integrated into a single database. Filtering can also aid in anomaly detection (for example, identification of samples that may have been subject to powder contamination).

4. Preserve relationships between data In addition to storing the data, the database should log information about how the pieces of data relate to one another. For instance, a specific lot of powder should be linked to all the builds that originate from it.
5. Provide basic tools for data display and analysis From the perspective of functionality, the database should enable the user to:

(a) Perform a search based on attribute values. The user should be able to browse for a specific attribute (or attributes), specify a range of attributes to search within, generate a list of materials that meet specific criteria, and organize the search results into a data sheet.

(b) Create a comparison report. After performing a search, the user should be able to create a comparison table displaying records and properties of interest.

(c) Display data as a function of a specific parameter. The user should be able to create $x-y$ plots of numeric attributes against one another (Ashby charts), select which attribute will be plotted on each axis, control the scale of the data, and view the data in a tabular form if desired. The ability to display data in this manner enables graphical investigation of patterns/trends and phenomenological relationships.

6. Export data to other programs The database's internal display and analysis tools (described in criteria 5) are intended to be rudimentary. The database should thus allow export of data to programs such as Excel, MATLAB, and SPSS, for statistical analysis and/or more sophisticated graphical display. The database must also be able to generate summary documents cataloging build parameters and testing results (as well as any other desired attributes the user may want to convert into report form).

7. Link to the work order system The database's collection capability should enable entry of data through an electronic work request or work order system. Linking data entry to a task order means that the database can be automatically populated as technicians and engineers build and test AM parts. This approach also creates an integration of effort among multiple organizations who play a role in AM (materials testing, damage tolerance assessment, metals engineering, environmental effects) and establishes a methodology (analogous to the weld traveler system commonly employed in welding production) that ensures a high degree of traceability for parts.

8. Accept inputs from multiple sources While initially the database will be populated exclusively with data from the AM group at NASA Marshall, as the tool evolves it will likely incorporate data from other (non-NASA) 
entities. The ability to catalog entries originating from vendors, contractors, academic institutions and other government agencies is aligned with the broader goal of using an informatics approach to reduce redundant research efforts and provide a single-source point for AM data.

9. Be broadly accessible, but mindful of data sensitivity Although one stated objective of AM database development is broad dissemination of information, the sources of the data and the application of the AM parts will necessitate robust access controls. The database will likely incorporate vendor and contractor data (generally classified as SBU) and many of the AM builds are for propulsion components (which fall under ITAR restrictions). For data originating from industrial round robin testing, the vendors providing the part must be aliased in some manner to further preserve their anonymity. A means to validate user credentials and control which users have access to which data will be a key component of any AM database.

10. High degree of compatibility The current state of research and development in the AM community is fragmented. Because research in AM tends to be "stovepiped" due to intellectual property and ITAR concerns, any database developed by NASA should be highly compatible with other (existing and future) databases. Design of a compatible database architecture will enable data mining across platforms, permit the export of data from other databases into the NASA system (or, conversely, the extraction and export of NASA data to other platforms), and pave the way for a single-source AM database.

11. Real-time status monitoring The database should allow credentialed users the ability to track the AM parts or test data through the various processes that are required for the AM parts to be built, tested and analyzed and to receive custom, automated notifications through the throughout the AM process. This will facilitate logistics and improve the efficiency and throughput rate of the AM process.

12. Extensibility The links between process, structure, and properties are not well understood for AM and it is not clear what particular process parameters should be included in the database. As researchers learn more about various processes and their effects on various materials, inputs may change. The database should evolve with the $\mathrm{AM}$ process and as such requires a flexible architecture that can accommodate change.

The characteristics outlined above are believed to be those which will yield the type of database that is of maximum benefit to the AM user, research, and standards development communities.

\section{Development of the pilot database and usage}

A precursor to the AM database development effort at NASA focused on the selective laser melting data from additive manufacturing builds done at MSFC. Organized in a basic spreadsheet format, this document compiled data for SLM builds of Inconel 718, CoCr, 316L SS, Ti-6Al-4V, and a few other materials (although the bulk of the data is for Inconel). Attributes captured include the build parameters (laser power, scan speed, trace width, layer thickness, etc.), material conditioning (HIP, solution, aging, and/or surface treatments), test environment (pressure, humidity, temperature, and composition), tensile results (for the test specimens which accompany the build, as most AM builds themselves do not undergo destructive testing), fracture toughness, and fatigue. Not all records had complete data.

Software engineers in the NASA Materials and Processes Laboratory undertook the task of converting the existing SLM summary document into a database format. These data, which initially populated the AM database and served as the pilot for future efforts, accelerated the path to a minimally viable AM database tool that was deployed for testing purposes and to solicit feedback from the NASA user community. The first critical trade in database development was the choice of a host platform to house the SLM data (and ultimately the full-scale AM database). There are multiple data management softwares which support materials selection and provide access to material design data across numerous academic institutions, government agencies, and industry. Ultimately Athena, a NASA-developed software platform, was judged more effective for developmental data. The use of Athena will enable NASA to maintain full control over the development and future customization of the AM database. A system of access controls for sensitive data also already exists in Athena. Since Athena also hosts NASA's material data repository MAPTIS, there is an opportunity to fully integrate validated data in the AM database with MAPTIS through this platform. Athena provides robust controls over the flow of data entry and readily links to the work order system to facilitate automatic database population. Overall, the ability to provide broad access to data (yet still implement the controls required to preserve sensitivity), customize database structure, and integrate with MAPTIS and the internal work order system drove the choice of Athena over commercial off the shelf softwares.

Data from SLM builds of SLM 718 were used to construct a pilot database that could be deployed to analysts for testing and feedback. This pilot database is part of the Athena platform and has some of the search, filtering, segregation and analysis capabilities identified in the previous section. Because the database is within Athena, NASA will be able to exercise control over future feature development 
Fig. 1 Overview of additive manufacturing database organizational schema

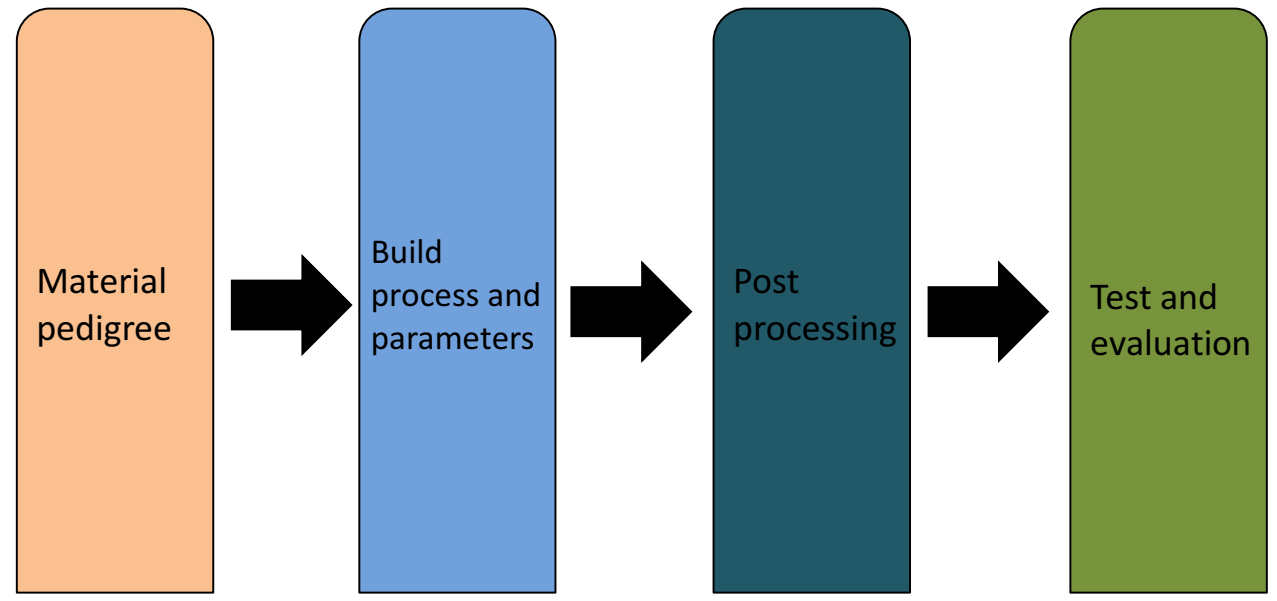

and customization activities. The initial data schema is detailed in Fig. 1. The schema is similar to that for other databases cataloging material property information, but is intended to also capture the large number of process variables and the complexities of the AM process. Metallic Materials Properties Development and Standardization (MMPDS), other commercial data sources, and standards provided a good foundation for identifying the pertinent information that must be captured in the database.

A critical aspect of developing the AM database was identification of the attributes and related organizational scheme that would best facilitate exploration of relationships between significant test input factors and outputs. The ultimate goals of the informatics approach are to:

1. guide development of mechanical properties to be used in design for AM,

2. further the technological maturity of the AM process as it applies to aerospace hardware,

3. inform approaches for quality control and certification for a given AM process.

From the analyst's perspective, an AM database should fundamentally be user friendly, handle data input/output flow quickly, be accessible to multiple users simultaneously, perform custom filtering and sorting of data based on custom search criteria, save those criteria sets for future analyses, and be secure. Currently the NASA AM database contains thousands of individual test records and approximately 200 test attributes. These attributes include pedigree information, manufacturing parameters, heat treatment and surface finish protocols, sample machining parameters, testing conditions, and mechanical test results. The scale of the dataset requires a robust database structure to efficiently store information and allow for scalability (continued growth of the amount of data), maximize the knowledge captured for each record, and provide an efficient way to interrogate the dataset. One fundamental goal must be to establish a system that allows individuals to easily and effectively contribute data to the database (e.g., engineers would provide the initial requirements, the AM build operator would provide manufacturing parameters and build records, an engineer or technician would provide the heat treatment information, and the test engineer would provide details of testing and results).

The list of attributes the database must capture, listed in Table 1, conveys the complexity of the dataset. These attributes were identified with the specific intent of developing a prototypical AM database for Inconel 718 round tensile samples to evaluate process/property relationships and provide initial feedback. Depending on the attribute, data can consist of binary (yes/no) inputs, lists of choices, alpha-numeric entries, and informational files and pictures. The database captures information arising from the pedigree of the powders used in the build (column 1), the build itself (column 2), subsequent post-processing/heat treatment if performed (column 3), and test conditions, apparatus, and results, if any (column 4). Not shown in this flow process are the details of any post-process machining or surface treatments.

The most critical test results for determining optimal build input factors are those from mechanical tests such as density, tensile test, fracture toughness tests, and fatigue tests. Because of the complexity of the materials and their use in multiple designs with widely varying requirements, the ultimate outcome of interest is what settings produce an acceptable set of material properties for a given application. The ability to sort and filter the AM database data in a customized manner and product custom, user-defined reports is critical to evaluating the effects of the various build input factors on properties. Storage and organization of the data in this manner facilitates future work on baseline property development and defining allowables for AM machines, materials, and processes. 
Table 1 Summary of data in AM database by category

\begin{tabular}{|c|c|c|c|}
\hline Material pedigree data & Build process and parameters & Post-processing & Specimen testing $^{\mathrm{c}}$ \\
\hline Material name & AM process & Heat treat combination number ${ }^{\mathrm{b}}$ & Type of test \\
\hline Powder supplier & Machine vendor & & Environment (temperature, humidity, media) \\
\hline Powder lot number & Machine model number & & Test date \\
\hline Material certification & Core laser power & & Test operator \\
\hline Certification date & Core scan speed & Heat treatment success status & Test location \\
\hline \multirow{2}{*}{$\begin{array}{l}\text { Chemistry report (powder } \\
\text { morphology, size) }\end{array}$} & Outside contour laser power & & Test specification \\
\hline & Outside contour scan speed & & Specimen drawing \\
\hline \multirow[t]{11}{*}{$\begin{array}{l}\text { Build origin (internal or } \\
\text { outside vendor) }\end{array}$} & Inside contour laser power & & $\begin{array}{l}\text { Measurements (diameter, gage length, failure } \\
\text { location) }\end{array}$ \\
\hline & Inside contour scan speed & & $\begin{array}{l}\text { Instrumentation (extensometer and load cell } \\
\text { information) }\end{array}$ \\
\hline & Trace width & & $\begin{array}{l}\text { Test machine parameter (e.g., displacement } \\
\text { rate) }\end{array}$ \\
\hline & Build layer thickness & & \\
\hline & Scan path & & Test data records and reports \\
\hline & Build file & & $\begin{array}{l}\text { Any additional reports: metallography, trend } \\
\text { analysis, chemical analysis }\end{array}$ \\
\hline & $\begin{array}{l}\text { Screen shot of build orientation } \\
\text { and relation to other parts in the } \\
\text { build }\end{array}$ & & \\
\hline & Machine log files & & \\
\hline & Photographs of parts after print & & \\
\hline & Continuous build status $^{\mathrm{a}}$ & & \\
\hline & Notes & & \\
\hline
\end{tabular}

${ }^{a}$ Indicates whether build was interrupted

${ }^{b}$ Links to a reference file with information regarding the specifics of the heat treatment regimen

${ }^{\mathrm{c}}$ List of attributes that provide information regarding the testing of specimens. Test information is specific to the test type (round tensile, notched tensile, hardness, fracture, density, high cycle fatigue, etc.)

Properties reported in the database generally originate from test reports based on evaluation of witness coupons (typically round tensiles specimens or density blocks). This testing is often performed in-house by the mechanical test group, or, in situations where in-house testing capabilities do not exist or the number of specimens is too large to process effectively given laboratory support of other projects, at external facilities. Tests are generally always conducted in accordance with relevant ASTM standards. All test reports associated with a build (and in many cases the raw data) are also archived in the AM database.

Factors which influence properties are also cataloged: powder characteristics, powder chemistry, machine the parts or specimens were built with, build parameters, details (times and temperatures) of stress relief/hot isostatic pressing/heat treatment regime, test environments and conditions (temperature, amplitude and number of cycles for fatigue test, loading, etc.). For powder characteristics and powder chemistry, these data may originate from vendor-supplied data or from in-house analysis. Linking the aforementioned factors with material outcomes facilitates more efficient data mining and exploration of process/property relationships to optimize manufacturing for a given application. Cataloging this additional information about initial material characteristics and build conditions alongside outcome data also provides key insight into the underlying physics of selective laser melting processes. In this way, experimental data can be exploited to provide validation for physics-based process models or point the way toward sounder concepts, accelerating materials development by transitioning AM processes from skill based to knowledge based.

The level of variability in machines and processes can also be assessed using the database by filtering. For example, one study segregated specimens from two different machines (from the same manufacturer) that were built at identical process parameters and subjected to the same post-processing regime. These specimens exhibited statistically significant differences in tensile strength, elastic modulus, and fracture elongation [9]. Similar analyses of 
machine variability have indicated that material properties are specific to not only build parameters, but the equipment the specimens are produced on. These type of analyses that isolate the effect of a single variable on material outcomes, easily facilitated by use of the database, can be used to inform development of AM-specific specifications and calibrate expectations for achievable properties under a wide variety of conditions. Experimentally intensive screening experiments to determine which variables influence material properties and the degree of this influence may be reduced or in some cases eliminated altogether by use of existing data archived in the AM database. Filtering can be exploited to evaluate the impact of any combination of machine, material, test condition, or processing variable on material outcomes, potentially reducing the need for large design of experiments. Exploration of these process/property relationships through filtering (e.g., what is the effect of powder morphology on surface roughness for a particular build parameter) represents typical queries to the database.

The database can also be used to facilitate manufacturing processing optimization by informing selection of process parameters to achieve desired properties. Data can also inform designer expectations regarding characteristic properties for material/processing combinations and assist in identification of potential problems with machines or processes. In one instance, data from the database were used to pinpoint the source of anomalous material performance for a set of builds (contamination as a result of powder changeout). Where appropriate and restrictions on data policy permit, the database can also be used by other organizations and compared against their own data archives. This information sharing, when possible, reduces redundant efforts and is key to development of standards for AM as well as improvement of process models.

While currently the database catalogs material development data from "for space" applications (mainly propulsion components), eventually the scope of data may expand to include "in-space" manufacturing capabilities. There are currently two fused deposition modeling (FDM) 3D printers on board the International Space Station (ISS). While data from material testing of specimens from the first printer are archived in MAPTIS, builds from in-space additive manufacturing are not part of the NASA AM database at the time of this writing.

\section{Summary and conclusions}

While informatics is an established approach for materials selection, it has historically been underutilized by the AM research community. Informatics has the potential to address the technical challenges encountered in AM development work by providing a knowledge base that can:

- Facilitate development of standards and protocols which will provide a clear path to flight certification for additively manufactured hardware and reduce risk associated with the use of AM parts

- Enhance process monitoring of AM by establishing a flow of data entry that allows data consumers to follow a part from build through inspection

- Create validated data that will enhance fundamental understanding of AM processes, enable better predictive models (the degree of uncertainty in models is directly correlated to the quality of the data) and yield higher quality products

- Reduce redundant research efforts in government and industry through the broad dissemination of data (when ITAR and proprietary data concerns permit)

- Enable broader use of AM technology and increased confidence in products.

The NASA MSFC approach to AM database development was to initially construct a database populated with the SLM build and test data. This pilot database, hosted on the NASA-developed Athena software platform which also houses MAPTIS, was tested by NASA consumers of AM data. Feedback from these users informed subsequent refinement of database structure and capabilities. A parallel ongoing effort seeks to identify attributes of additive manufacturing processes (including but not limited to powder characteristics, build parameters, mechanical test data, post-process heat and surface treatment protocols) that are of maximum benefit to analysts exploring relationships between process and properties. This work will determine the fields for data entry and (to some extent) the filtering scheme. The final database structure will be designed to enable efficient and value-added mining of data to maximally support analysis efforts. Ultimately, data entry will be linked, as much as possible, to the work order system to enhance traceability, reduce manual user input errors, and enable process monitoring in a manner similar to the wellestablished weld traveler system.

At the time of this writing, the preliminary AM database has been deployed in MAPTIS and is currently used by NASA personnel to facilitate mining and analysis of internal AM data for SLM of In718. Looking toward the future, the AM database should be able to evolve with the AM process and accommodate the diverse research directions AM technology may take in the coming years, both at NASA and in the broader AM community. Integration across multiple platforms to support fluid export and import of data from similar informatics efforts in government and industry is key to enabling this evolution. 
The AM database development effort is aligned with a number of similar data management efforts in government and academia. The overall trend toward so-called "big data" initiatives is anticipated to continue as information technology and high-performance computing remains a crossagency scientific priority [1]. The 2010 Decadal Survey called for creation of "robust databases that could be used for extramural scientists to address research questions" [10]. Data management programs established in response to these recommendations, which include the Materials Genome Initiative (MGI) from NIST and NASA's open source data initiative for International Space Station science, seek to accelerate scientific discovery by making data as widely available as possible while still providing appropriate access restrictions. The goals of an AM database (to provide high-quality data, expedite discovery and the path from research to product, and serve as an enabler for data dissemination and standards development) are shared with many diverse informatics programs (either proposed or existing) in the science community. America Makes and DARPA have awarded major projects to Penn State Applied Research Lab and Army Research Lab to develop informatics approaches for AM. Similarly, the AMAZE project in Europe has partnered with the commercial software company GRANTA to develop database schema for AM [11]. Leveraging the resources and expertise of the entire AM research community is critical to transition the technology from skill based to knowledge based and accelerate AM's use in aerospace and other industries where it has the potential to reduce costs and lead times.

Informatics serves as the foundation that can facilitate full characterization of AM materials and create increased confidence in additively manufactured products, thereby enabling broader use of the technology. Though informatics is a well-established approach for materials selection, it has not yet found wide use in the AM research community. An informatics approach to AM data management enables the AM community to continue to push the boundaries of manufacturing technology while negotiating a careful and healthy balance between risk, schedule, performance and affordability.

\section{References}

1. Deese BC, Holdren JP (2014) Office of Management and Budget and Office of Science and Technology Policy. Memorandum for the Heads of Executive Departments and Agencies: Science and Technology Priorities for the FY 2016 Budget

2. United States. Executive Office of the President National Science and Technology Council (2012) A National Strategic Plan for Advanced Manufacturing. http://www.whitehouse.gov/sites/ default/files/microsites/ostp/iam_advancedmanufacturing_strategicplan_2012.pdf. Accessed Aug 2014

3. About America Makes. America Makes. https://americamakes. us/about/overview. Accessed 8 Aug 2014

4. Catts T (2013) GE Printing Engine Fuel Nozzles Propels \$6 Billion Marke. Bloomberg. http://www.bloomberg.com/news/articles/2013-11-12/ge-printing-engine-fuel-nozzles-propels-6-billion-market. Accessed Aug 2014

5. Wells, D. (2016) Key Quality Assurance Metrics in Additive Manufacturing. NASA Quality Assurance in Additive Manufacturing: A Workshop on Assuring AM Product Integrity. Workshop at California Institute of Technology, Pasadena, CA. http:// ntrs.nasa.gov/archive/nasa/casi.ntrs.nasa.gov/20160013359.pdf

6. United States. Department of Commerce. National Institute of Standards and Technology (2013) Measurement science roadmap for metal-based additive manufacturing. Energetics Incorporated. http://www.nist.gov/el/isd/upload/NISTAdd_Mfg_ Report_FINAL-2.pdf. Accessed Aug 2014

7. United States. National Aeronautics and Space Administration (2008) Standard materials and processes requirements for spacecraft. http://prod.nais.nasa.gov/eps/eps_data/122687OTHER-001-008.pdf. Accessed Aug 2014

8. Kraft R, Tracy M, Kim H (2013) NASA Tests Limits of 3-D Printing with Powerful Rocket Engine Check National Aeronautics and Space Administration Release 13-260. http://www.nasa. gov/press/2013/august/nasa-tests-limits-of-3-d-printing-withpowerful-rocket-engine-check/\#.VsjuCsfp51I. Accessed Aug 2014

9. Prater $\mathrm{T}$ et al (2015) Study of machine variability and progressive heat treatment in selective laser melting of Inconel 718. In: Proceedings of the 62nd JANNAF Propulsion Meeting. Nashville, pp 1-5

10. National Research Council of the National Academy (2011) Recapturing a future for space exploration: life and physical sciences research for a new era

11. Lu Y, Choi S, Witherell P (2015) Towards an integrated schema design for additive manufacturing: conceptual modeling. In: Proceedings of ASME 2015 International Design Engineering Technical Conferences \& Computers and Information in Engineering 